Int. J. Speleol. 9 (1977), pp. 65-71.

\title{
Stenasellus escolai n. sp., Crustacea Isopoda Asellota des eaux souterraines d'Espagne méridionale
}

\author{
par
}

Guy MAGNIEZ*

\begin{abstract}
SUMMARY
Stenasellus escolai n. sp., Crustacea Isopoda Asellota from the subterranean waters of Southern Spain.

This new species was captured in the phreatic waters of Guadalquivir valley, where it lives together with another, very small-sized Stenasellid: St. bragai Magniez, previously described.

It belongs to the phyletic line of St. breuili Racovitza, which colonizes the subterranean waters of the Iberic peninsula.
\end{abstract}

ORIGINE DU MATÉRIEL: Ce nouveau Sténasellide a été capturé par Monsieur Claude Bou, en août 1972, au cours de prospections des eaux souterraines d'Espagne méridionale par le procédé des sondages tubés Bou-Rouch. La station est située surr le Guadalquivir, à environ $10 \mathrm{~km}$ en amont du confluent du fleuve avec le Guadiana Menor, un peu en aval du barrage supportant la route de Torreperogil à Peal-de-Becerro, province de Jaèn (renseignements aimablement communiqués par mon collègue $\mathrm{Cl}$. Bou que je remercie de la confiance qu'il me témoigne en mettant ce matériel à ma disposition). Nous dédions cette forme au Dr. O. Escolà, de Barcelone, en raison de ses importantes contributions à la biospéléologie espagnole.

LOT CAPTURÉ: $3 \hat{o}$ adultes de 4,5; 4,7 et $5 \mathrm{~mm}$; 19 de $4 \mathrm{~mm}$ immature (sans oostégites); 1 jeune de $1,5 \mathrm{~mm}$ sans péréiopodes VII (1er stade libre); 2 jeunes de $1,7 \mathrm{~mm}$ à péréiopodes VII rudimentaires (2ème stade libre) et 1 jeune de $1,9 \mathrm{~mm}$ à péréiopodes VII parfaits, mais plus courts que les VI (3ème stade libre). Les dimensions de ces trois stades larvaires libres, correspondant aux trois premières intermues postmarsupiales, sont donc pratiquement identiques à celles relevées chez Stenasellus virei.

* Laboratoire de Biologie Animale et Générale, Université de Dijon, 6, Bd. Gabriel, 21000 Dijon, France. 


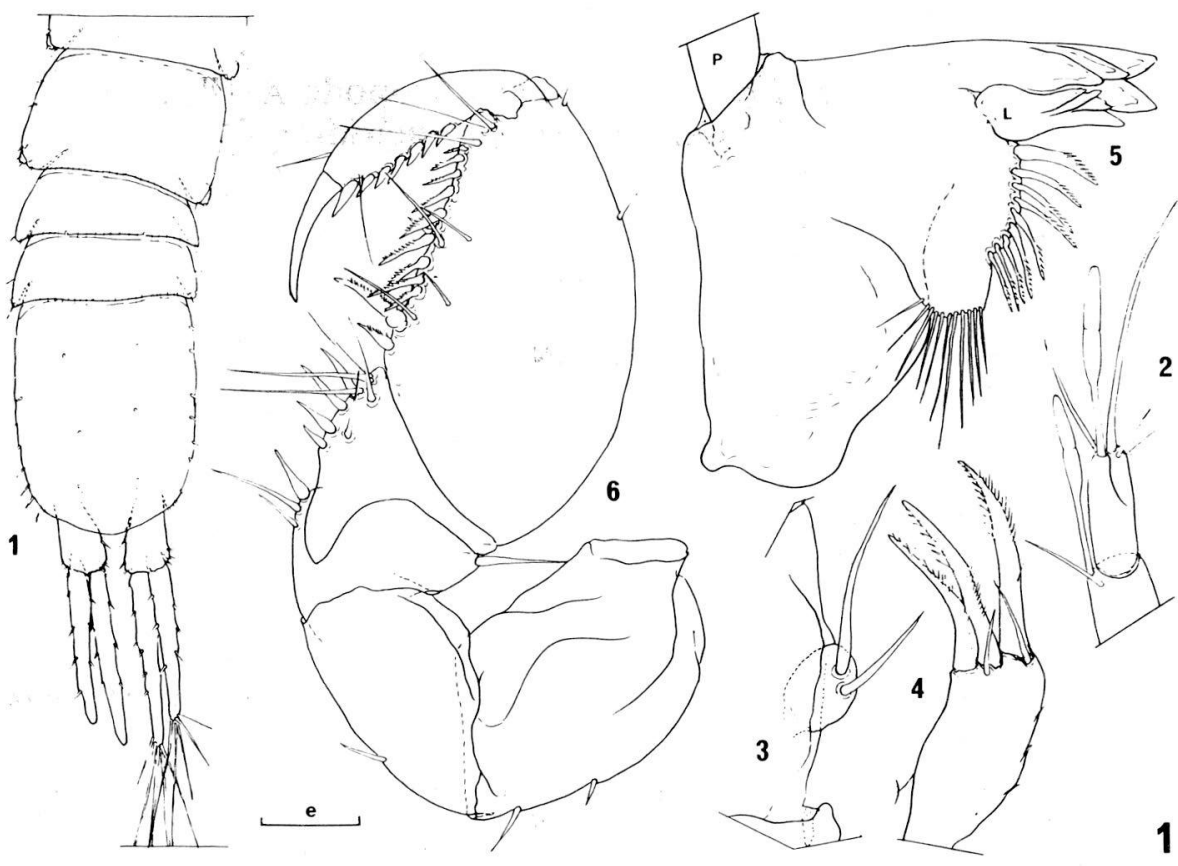

Figures I:

1. Péréionite VII, pléon et uropodes de la $q$ de $4 \mathrm{~mm}$; $\mathrm{e}=333 \mu$.

2. Extrémité d'une antennule du $\hat{\sigma}$ de $5 \mathrm{~mm}$; $\mathrm{e}=50 \mu$.

3. Article III de la hampe et squama d'une antenne du même; e $=50 \mu$.

4. Lobe interne de la maxillule gauche du même; $\mathrm{e}=33 \mu$.

5. Mandibule gauche du même $(\mathrm{P}=$ palpe, supprimé; $\mathrm{L}=$ lacinia mobilis $) ; \mathrm{e}=50 \mu$.

6. Péréiopode I gauche du même; $\mathrm{e}=100 \mu$.

CARACTÉRES GENERAUX: St. escolai $\mathrm{n}$. sp. est un Stenasellus de taille moyenne, dont les $q$ adultes devraient atteindre quelques 7-8 $\mathrm{mm}$. L'aspect général rappelle celui de $S t$. virei virei: téguments bien indurés, corps grêle (largeurs respectives de la tête, des péréionites I, IV, VII et du pléotelson: 640, $650,700,680$ et $620 \mu$, chez la $\varsubsetneqq$ de $4 \mathrm{~mm}$ ), mais appendices robustes, avec une chétotaxie générale bien développée.

Céphalon subtrapézoïdal, plus large que long, à bord rostral bien concave. Régions pleurales des péréionites étroites, coxopodites des péréiopodes II-VII aussi développés que ceux de $S t$. virei et débordant légèrement les péréionites (fig. 1, 13). Pléonites I et II bien développés en longueur. Pléotelson allongé, à pointe caudale effacée (fig. 1).

APPENDICES CÉPHALIQUES: Fouet de l'antennule (fig. 2) comptant jusqu'à 9 articles avec 6 lames olfactives de 95-100 $\mu$ sur les distaux (fouet de 2 

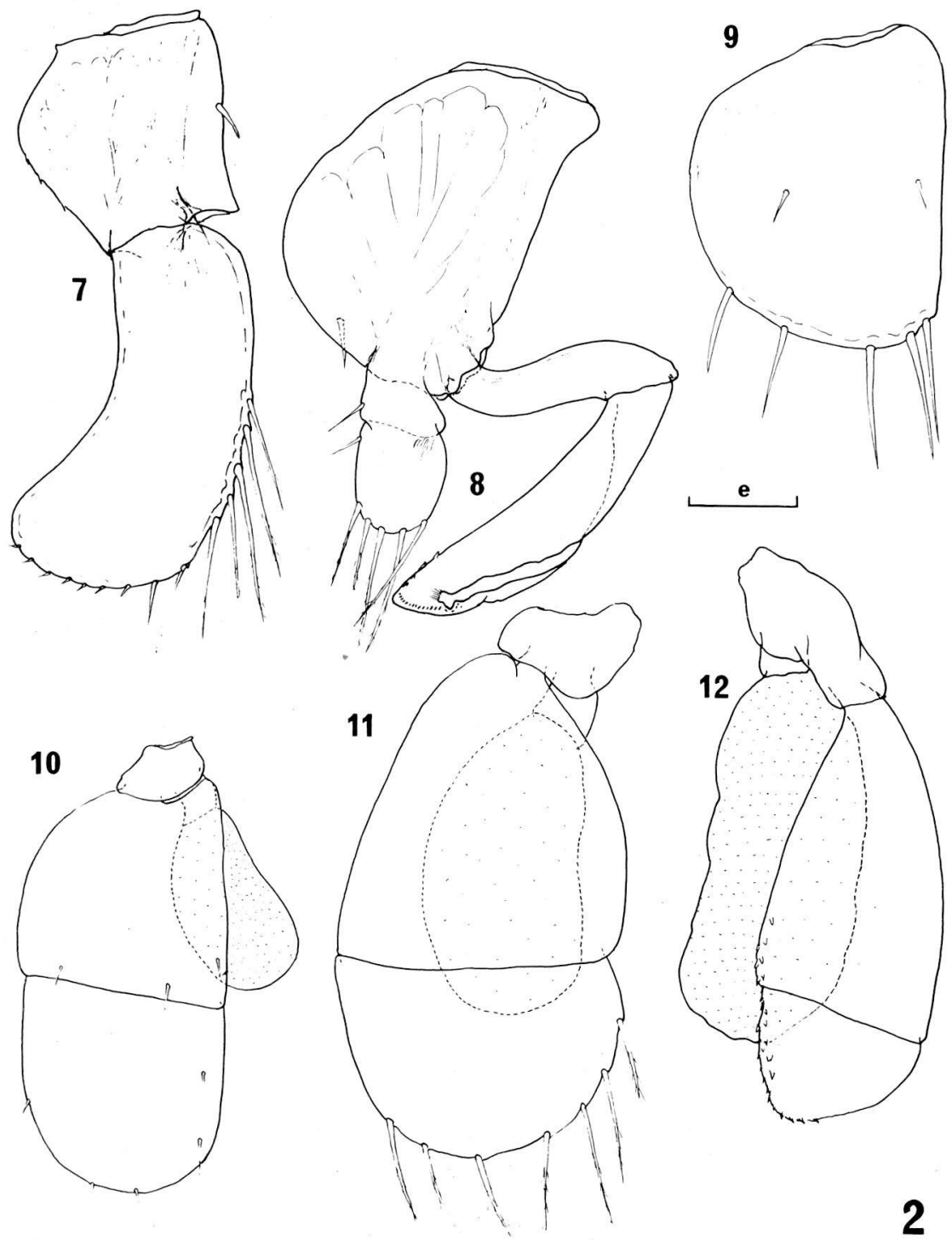

Figures II:

7. Pléopode I gauche du $\hat{\delta}$ de $5 \mathrm{~mm}$; $\mathrm{e}=100 \mu$.

8. Pléopode II gauche, face tergale, du même; $\mathrm{e}=100 \mu$.

9. Pléopode II droit de la $q$ de $4 \mathrm{~mm}$; e $=100 \mu$.

10. Pléopode III droit du $\hat{O}^{+}$de $5 \mathrm{~mm} ; \mathrm{e}=200 \mu$.

11. Pléopode IV droit du même; $\mathrm{e}=100 \mu$.

12. Pléopode $\mathrm{V}$ gauche du même; $\mathrm{e}=100 \mu$. Les endopodites des pléopodes III, IV et $\mathrm{V}$, charnus et respiratoires, sont figurés en pointillés. 

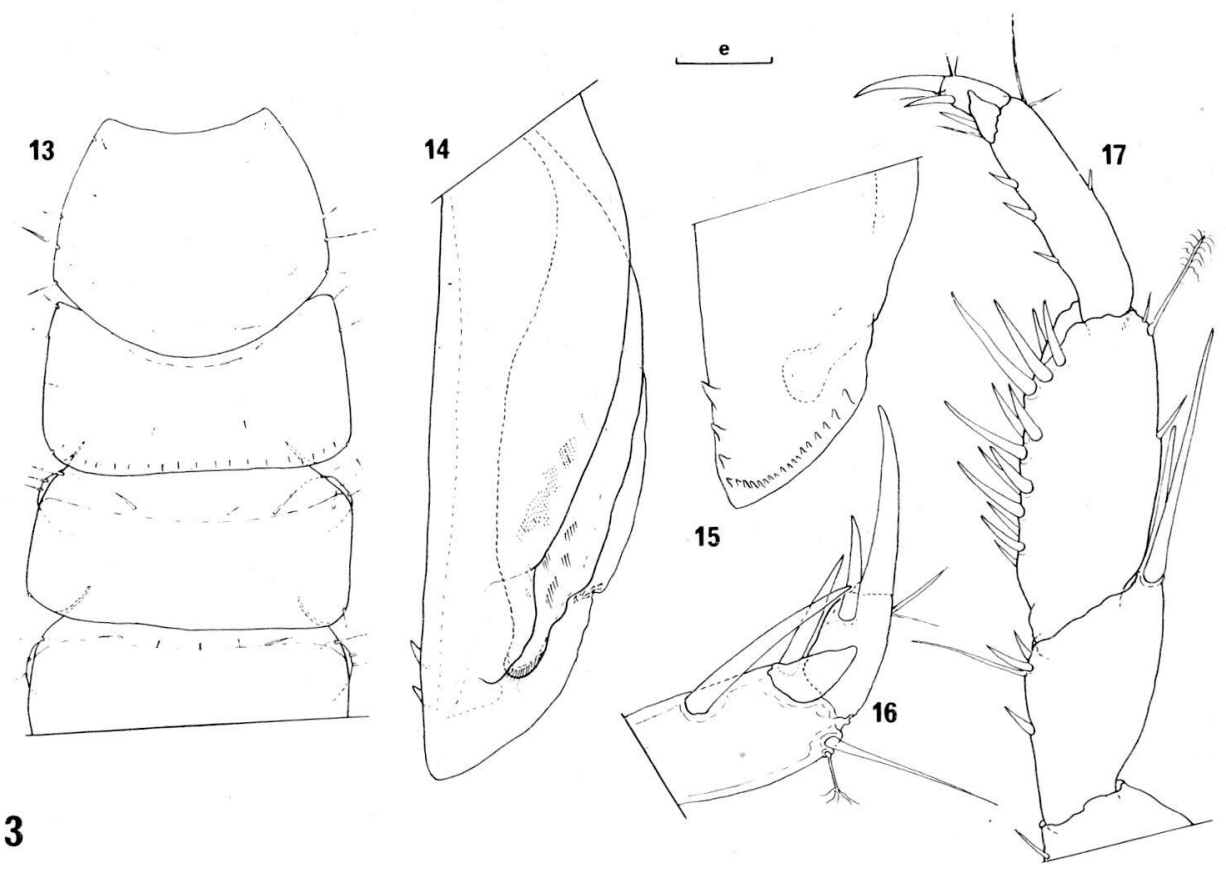

3

Figures III:

13. Céphalon et premiers péréionites, face dorsale, de la $q$ de $4 \mathrm{~mm} ; \mathrm{e}=200 \mu$

14. Extrémité de l'endopodite, face tergale, du pléopode II gauche du $\hat{\delta}$ de $5 \mathrm{~mm}$. Les sétules tapissant la paroi interne et bordant l'orifice efférent ont été schématisées; $\mathrm{e}=33 \mu$.

15. Le même, face sternale, montrant l'armature de denticules chitineux distaux. L'orifice efférent, tergal, est indiqué en pointillés; $\mathrm{e}=33 \mu$.

16. Extrémité du péréiopode VII gauche de la $\bigcirc$ de $4 \mathrm{~mm}$; $\mathrm{e}=50 \mu$.

17. Péréiopode $\mathrm{V}$ gauche du $\hat{\sigma}$. de $5 \mathrm{~mm}$, montrant la puissante armature sternale du carpopodite; $\mathrm{e}=100 \mu$.

articles, avec une seule L.O. chez le jeune au premier stade; 3 articles + 1 L.O. au deuxième stade et 4 articles +2 L.O. au troisième).

Antennes atteignant la moitié de la longueur du corps, squama bien développée, portée par le troisième article de la hampe (fig. 3) et munie de 2 tiges lisses; fouet de 25-27 articles chez le $\hat{\delta}$ adulte, 22-23 chez la $\uparrow, 10$ seulement aux ler et 2ème stades et 11 au 3ème stade.

Mandibules typiques, massives (fig. 5) avec palpe triarticulé normal; article distal munı d'un peigne de 8 tiges lisses croissant distalement (la distale atteint 120-130 $\mu$ ).

Maxillules normales, à lobe externe portant 11 fortes épines barbelées distales de 30-50 $\mu$, disposées sur deux rangs et quelques sétules sur la marge interne; lobe interne (fig. 4) avec 3 fortes tiges rameuses et 2 soies simples sur la marge distale; marge externe portant quelques sétules. 
Maxilles trilobées normales; lobes externe et moyen portant chacun une rangée distale de 7 lames falciformes, dentelées sur leur marge interne, de longueur décroissant de l'extérieur $(70 \mu)$ vers l'intérieur $(20 \mu)$; lobe interne entier, portant distalement une quinzaine de tiges rameuses polymorphes (30-55 $\mu$ ), implantées sur deux rangs, ainsi que de fines soies sur la marge interne.

MAXILLIPĖDES: Typiques de Stenasellidae, sans épipodite; endite avec 2 crochets rétinaculaires.

PÉRÉOPODES I: Assez semblables à ceux de St. virei (fig. 6). Propodite bien renflé, à marge sternale fortement armée de lames dentelées; marge interne du dactylopodite munie d'une rangée continue de lames simples.

PÉREIOPODES II Ȧ VII: Robustes et assez trapus, à chétotaxie bien développée, surtout au niveau de la marge sternale des carpopodites (fig. 17). Dactylopodites avec une seule épine sternale. Longueurs respectives des péréiopodes I-VII chez le $\delta$ de $5 \mathrm{~mm}: 1,41-1,47-1,49-1,45-1,59-1,87$ et 2,20 $\mathrm{mm}$. Longueurs relatives à celle de P.II: $0,96-1-1,014-0,98-1,08-1,27$ et 1,49. Une paire de papilles génitales longues et grêles insérées à l'angle interne des coxopodites VII chez les $\delta$.

PLEOPODES II $q$ : Petits et indépendants (fig. 9), à marges externe et distale régulièrement courbes.

PLEOPODES I $\hat{\sigma}$ : Protopodite subtrapézoïdal avec un crochet rétinaculaire sur la marge interne. Rame longue et étroite, à marge externe très concave. Une rangée continue de tiges plumeuses sur la marge interne-distale et de sétules sur la marge distale (fig. 7).

PLEOPODES II $\delta$ : Protopodite subquadrangulaire avec une forte épine submarginale sternale externe (fig. 8). Exopodite biarticulé avec marge distale du second article munie de longues soies plumeuses. Endopodite avec article proximal long et grêle; article distal falciforme armé distalement d'une rangée sternale d'épines (20 environ), décroissant vers l'intérieur (fig. 15). Orifice efférent subterminal-tergal, petit et à marge garnie de fines soies faisant saillie à l'extérieur. Parois de la gouttière interne garnies de rangées de sétules dirigées distalement (fig. 14).

PLEOPODES III: Exopodite (opercule) allongé; endopodite charnu, très petit (fig. 10).

PLEOPODES IV: Exopodite lamelleux à suture non oblique; article distal muni de longues soies marginales plumeuses (fig. 11); endopodite petit et charnu.

PLEOPODES V: Exopodite induré, biarticulé, en massue, plus long que l'en- 
dopodite; une aire glandulaire couverte d'écailles cuticulaires sur la marge interne-distale. Endopodite charnu arqué, respiratoire comme les endopodites III et IV (fig. 12).

UROPODES: Biramés normaux, pratiquement aussi longs que le pléotelson (fig. 1).

\section{REMARQUES:}

1. Les détails de structure (épines distales sur l'organe copulateur, constitution des pléopodes I $\hat{\delta}$, des pléopodes IV et V) montrent que St. escolai appartient à la lignée ibérique de St. breuili (cf. Magniez 1974a, p. 42-43), qui comprend aussi St. galhanoae Braga de puits de l'Algarve et St. bragai Magniez du sous-écoulement du Guadalquivir.

2. St. escolai n. sp. et St. bragai vivent ensemble dans le même biotope: la nappe fluviale du Guadalquivir. Le fait que les deux espèces aient été capturées ensemble en 1972 et que tous les stades de l'une et l'autre soient représentés dans le lot provenant d'un même pompage indique qu'il y a cohabitation parfaite entre elles, peut-être dans des interstices de tailles différentes, au sein des alluvions (cf. Magniez 1974b, p. 148, fig. 22). Jusqu'à présent, trois cas de cohabitation de 2 espèces de Sténasellides ont été signalés: Magniezia africana (Monod) + Parastenasellus chappuisi (Remy) dans un puits de Man (Côted'Ivoire), M. guineensis (Braga) + P. chappuisi dans un puits de Gabu (Guinée-Bissau) et Mexistenasellus wilkensi $+M$. parzefalli Magniez dans une grotte du Mexique. A chaque fois, l'association comprend une espèce robuste et fortement armée, dont ont peut penser que les moeurs carnassières sont développées et une seconde espèce plus faible, aux péréiopodes I grêles, sans doute plus polyphage. L'association de la nappe du Guadalquivir ne fait pas exception, puisque nous y trouvons une forme robuste aux péréiopodes I puissants (fig. 6): St. escolai n. sp., accompagnant une forme naine et gracile: $S t$. bragai (Magniez 1976, fig. 5-6).

3. Dans une publication précédente (Magniez 1974a), j'ai noté (p. 43) la découverte de St. escolai n. sp., ainsi que sa localisation géographique (p. 48, fig. 10, $\mathrm{n}^{\circ} 25$, Stenasellus sp. nov. 2).

Manuscrit terminé en octobre 1975.

\section{RESUME}

Cette nouvelle espèce à été capturée dans la nappe fluviale du Guadalquivir où elle vit en compagnie d'un autre. Sténasellide de très petite taille: St. hragai Magniez, précédemment décrit. Elle appartient à la lignée de Stenasellus hreuili Racovitza, répandue dans les eaux souterraines de la péninsule ibérique. 


\section{BIBLIOGRAPHIE}

BRAGA, J. M. 1962, Sur la distribution géographique des Stenasellus de la péninsule ibérique et description d'une espèce nouvelle de ce genre. An. Fac. Ciên. Porto, 44, 9-28.

MAGNIEZ, G. 1974a. Données faunistiques et écologiques sur les Stenasellidae (Crustaceae Isopoda Asellota des eaux souterraines). Int. J. Speleol., 6, 1-80.

MAGNIEZ, L. 1974b. Observations sur Stenasellus virei dans ses biotopes naturels (Crustacea Isopoda Asellota des eaux souterraines). Ibid., 6, 115-171.

MAGNIEZ, G. 1975. Observations sur la biologie de Stenasellus virei (Crustacea Isopoda Asellota des eaux souterraines). Ibid., 7, 79-228.

MAGNIEZ, G. 1976. Stenasellus bragai n. sp., Crustacea Isopoda Asellota des eaux souterraines d'Espagne méridionale. Ibid., 8, 285-290. 